\title{
Perfil da Clientela de um Ambulatório de Geriatria do Distrito Federal
}

\author{
Cristina Ramos Costa Linhares \\ Vera Lúcia Decnop Coelho ${ }^{1}$ \\ Renato Maia Guimarães \\ Ana Paula Martins de Campos \\ Nális Torres de Carvalho \\ Universidade de Brasilia
}

\begin{abstract}
Resumo
Há uma atenção crescente de clínicos e pesquisadores ao estudo do envelhecimento. É essencial um maior conhecimento sobre a população idosa, tendo em vista a melhoria de sua saúde, a capacitação de profissionais, e o planejamento de políticas públicas. Discute-se características sócio-demográficas e a pontuação na GDS de pacientes atendidos no Ambulatório de Geriatria do Hospital Universitário de Brasília (HUB). A amostra inclui 303 pacientes, com 71 anos (média), mulheres (67,3\%), até quatro anos de escolaridade $(74,4 \%)$, casadas $(49,8 \%$ ) ou viúvas (30,4\%). Enquanto 42,9\% vêm sós ao Ambulatório, 37,1\% são acompanhados pelos filhos. Embora a maioria dos idosos (63,3\%) não apresente indício de depressão pela GDS, 36,7\% deles têm algum índice de depressão. Informação acerca desses e de outros tópicos contribui para o planejamento de estratégias de assistência e para o aprimoramento de modelos de avaliação clínica nos serviços públicos de saúde.

Palavras-chave: Envelhecimento; idosos; ambulatório de geriatria; perfil da clientela.
\end{abstract}

Patients' Profile at a Geriatric Outpatient Service in Federal District, Brazil

\begin{abstract}
There is a growing attention of clinicians and researchers to the study of aging and the elderly. It is essential a better understanding of this population in order to allow an improve in their health, a better training of professionals, and an adequate planning of public health policies. The study presents sociodemographic characteristics and scores on the Geriatric Depression Scale of patients seen at the Geriatric Outpatient Unit, Brasilia University Hospital, Federal District. The sample includes 303 patients, with mean age of 71 years, women (67.3\%), up to four years of education (74.4\%), married (49.8\%)or widowed (30.4\%). Patients come to the Clinic by themselves (42.9\%)or with their children (37.1\%). Although most patients $(63.3 \%)$ do not present signs of depression, $36.7 \%$ of them present some index of depression. Information on these topics may help planing alternative health treatment strategies, and also improve clinical assessment for this population.

Keywords: Aging; elderly; outpatient geriatric service; patients' profile.
\end{abstract}

Muitos são os desafios a serem enfrentados no presente século. Os problemas relativos ao meio ambiente e a progressiva exclusão social constituem uma unanimidade entre os que visualizam a agenda dos próximos 100 anos. $\mathrm{O}$ envelhecimento populacional nem sempre é lembrado, mas é uma questão de igual magnitude.

O processo de envelhecimento é inerente à vida, constituindo um fenômeno complexo que afeta o sujeito humano de modo muito particular. Diferenças na experiência do envelhecimento se devem a fatores biológicos, psicológicos, sociais, e culturais que determinam a existência humana (Fernandes, 2000).

Mas o que delimita ser velho em nossa sociedade? Convencionou-se a idade de 60 anos como sendo o início da velhice nos países em desenvolvimento e 65 anos nos países desenvolvidos. De acordo com Veras (1994), três faixas etárias podem ser delimitadas: jovens idosos (pessoas

${ }^{1}$ Endereço para correspondência: Colina Bloco E 605, 70910 900, Universidade de Brasília, Brasília,DF.E-mail:vldc@unb.br entre 60 a 69 anos), meio-idosos (entre 70 e 79 anos), e idosos velhos, que abriga aqueles com idade acima de 80 anos.

Autores divergem sobre a partir de quando tem início o envelhecimento humano. Guimarães (1996) afirma ser um engano considerar que o envelhecimento começa no nascimento. $\mathrm{O}$ autor defende que até em torno de 30 anos o corpo humano é inundado de hormônios, responsáveis pelo desenvolvimento de características sexuais e de reprodução. A partir de então se inicia um processo gradual de perda orgânica, marco do envelhecimento.

Dados do Censo 2000 apontam que indivíduos com idade acima de 60 anos representam $8,56 \%$ da população brasileira, dos quais $55 \%$ são mulheres. A expectativa de vida da população brasileira foi estimada em 68 anos e 7 meses. Os homens vivem, em média, 64,8 anos e as mulheres, 72,6 anos; em média estas últimas têm, portanto, 8 anos a mais de vida (wnw.ibge.gov.br).

O envelhecimento populacional gera demandas em diversas áreas, sendo que na Previdência Social o gasto com aposentadorias é apontado como um grande fator 
do desequilíbrio atual das contas públicas. $\mathrm{Na}$ área da saúde a situação não é menos complexa, a despeito de não merecer a atenção devida. Cerca de $25 \%$ dos gastos da assistência hospitalar do Ministério da Saúde são consumidos por pacientes com 60 ou mais anos de idade, não obstante constituírem mais de $8 \%$ da população (Saúde do Idoso, 2002). Ainda assim, a assistência à saúde dos idosos é reconhecidamente deficiente. O episódio da Casa de Saúde Santa Genoveva, no Rio de Janeiro, é emblemático.

Com o aumento do número de idosos no Brasil e no mundo, estudos sobre essa população tornam-se cada vez mais necessários, como também a busca de capacitação profissional específica e um melhor planejamento das políticas de saúde pública. É sabido que à medida que se envelhece surgem doenças crônicas, dentre elas a hipertensão arterial e doenças osteoarticulares que contribuem, juntamente com maus hábitos de saúde, para a incapacitação funcional do idoso. A diminuição da capacidade funcional não está somente relacionada à doenças físicas; a demência e a depressão, por exemplo, podem gerar limitações importantes e alterar significativamente a qualidade de vida das pessoas. Outros modelos, além da assistência hospitalar e ambulatorial tradicional tornam-se, portanto, necessários. $\mathrm{O}$ atendimento domiciliar e o hospital-dia são exemplos de alternativas assistenciais a serem consideradas em nosso país.

O estudo do envelhecimento humano nem sempre foi objeto de interesse da Psicologia. Durante uma parte significativa do século XX pesquisadores concentraram esforços na compreensão da infância e adolescência. A concepção dos anos tardios de vida como um período exclusivamente de declínio e perdas pode ter contribuído para a manutenção de mitos e preconceitos acerca do envelhecimento (Almeida, 1999).

De acordo com Neri (1995), somente a partir dos anos 1950 a pesquisa sobre envelhecimento humano ganha força por razões diversas. Neste contexto, Erik Erikson contribuiu para impulsionar uma perspectiva de que o desenvolvimento se dá ao longo da vida (Papalia \& Olds, 2000). Assim, as formulações teóricas desse estudioso do desenvolvimento humano despertou em pesquisadores e clínicos uma preocupação com as transformações e crises vivenciadas nos anos tardios de vida.

Paul Baltes, formulando a perspectiva teórica do curso de vida, contribuiu para a consolidação da Psicologia do envelhecimento (Neri, 1995). Desconstrói-se, dessa forma, a imagem da pessoa idosa como decadente, e do envelhecimento como um processo unicamente de perdas (Linhares, 2002).
Atualmente, devido ao avanço nas teorias e pesquisas, pode-se falar em 'envelhecimento satisfatório', que depende da constante interação do indivíduo com o meio ambiente e do equilíbrio entre suas limitações e potencialidades, permitindo-lhe enfrentar perdas comuns nessa fase da vida, como aposentadoria, doenças e mortes. Para que o indivíduo envelheça bem, portanto, dependerá de suas chances para usufruir de condições adequadas de saúde, educação, moradia, alimentação, durante todo seu curso de vida. Acrescenta-se a isso, de acordo com Guimarães (1996), que o conhecimento do processo de envelhecimento poderá ajudar os idosos a viver com um bom nível de saúde.

Apenas a minoria dos indivíduos têm uma velhice patológica. Estudos em comunidade têm indicado que a prevalência dos transtornos mentais nos idosos varia de 15 a $30 \%$, o que significa que grande parte dessa população é saudável (Blay, 1989). Dentre os transtornos mais freqüentes no idoso, está a depressão. Estudos epidemiológicos demonstram que os quadros depressivos sofrem aumento de prevalência diretamente proporcional à idade (Aguiar \& Dunningham, 1993; Stoppe Jr. \& Louzã-Neto, 1999), o que sugere a importância de se dar atenção a este problema na população idosa.

Os quadros depressivos apresentados por idosos freqüentemente são caracterizados por aspectos clínicos particulares ou incomuns em relação à depressão apresentada por indivíduos jovens. Sintomas somáticos, dores crônicas, distúrbios do sono e apetite são importantes queixas apresentadas por idosos deprimidos. Isto dificulta a definição diagnóstica, já que esses sintomas podem fazer parte da realidade do mundo dos idosos cujo envelhecimento é normal (Stoppe Jr., Jacob Filho \& Louzã-Neto, 1994).

Segundo Blazer e Koening (1999), diversos fatores contribuem para a dificuldade em identificar-se casos de depressão em pessoas idosas: a depressão pode ser mascarada por alguma doença física ou até mesmo pela pseudodemência; as categorias dos atuais manuais de diagnóstico nem sempre se ajustam aos sintomas exibidos e relatados por pacientes idosos. $\mathrm{O}$ fato de algumas queixas comumente atribuídas ao envelhecimento constarem do repertório de sintomas depressivos, pode contribuir para o não reconhecimento de transtornos do humor em idosos. Esta foi um das hipóteses discutidas por Guimarães, FreireNeto, Costa, Silva e Moura (1997) para justificar a omissão do diagnóstico de depressão (leve, moderada ou grave) em $40 \%$ da amostra de pacientes internados em enfermarias cirúrgicas, que apresentavam sintomas depressivos em grau variado. 
No que diz respeito à avaliação sintomatológica de depressão no Brasil, O. P. Almeida e S. A. Almeida (1999) demonstraram recentemente que as versões brasileiras da Escala de Depressão Geriátrica (GDS) de 15 itens oferecem medidas válidas para o diagnóstico de Episódio Depressivo Maior, de acordo com os critérios do DSM-IV (APA, 1994) e CID-10 (WHO, 1992), além de constituírem medidas confiáveis da gravidade do quadro depressivo.

De acordo com Blay (1989), qualquer contato do indivíduo idoso com o sistema de saúde é um importante momento de se fazer a detecção de possíveis problemas psiquiátricos. Assim sendo, o ambulatório de Geriatria enquanto instância clínica representa não somente uma porta de entrada do idoso no ambiente hospitalar, mas igualmente uma importante fonte de informações sóciodemográficas e clínicas. Estudos que utilizem essa população e esse ambiente específico para coleta de dados constituem um campo promissor e essencial à área de saúde (Almeida,1999; Almeida, Garrido \& Tamai, 1998; Taddei \& cols., 1997).

\section{Objetivos}

Objetivo geral: Ampliar o conhecimento acerca da população idosa usuária do sistema público de saúde em nosso país, visando contribuir para o planejamento e implementação de propostas de assistência à saúde do idoso.

\section{Objetivos específicos:}

1) Identificar algumas das características sóciodemográficas de pacientes atendidos em um Ambulatório de Geriatria da rede pública do Distrito Federal;

2) Identificar a pontuação dessa clientela na Escala de Depressão Geriátrica (GDS);

3) Identificar aspectos a serem possivelmente incluídos na avaliação clínica de pacientes idosos ambulatoriais.

\section{Método}

Coletou-se informações referentes a pacientes atendidos no Ambulatório de Geriatria do Hospital Universitário de Brasilia (HUB), no período de março de 2000 a março de 2001. Os dados aqui analisados são provenientes da Avaliação Geriátrica Objetiva (AGO), que consta do prontuário dos pacientes. Este instrumento de coleta de informações na consulta médica foi desenvolvido para o ensino da Medicina Geriátrica no HUB e considera os sinais e sintomas mais prevalentes entre os idosos, priorizando a formulação de problemas e não apenas diagnósticos. $\mathrm{O}$ rastreamento de sintomas compatíveis com depressão é incentivado, adotando-se como instrumento a Escala de
Depressão Geriátrica (GDS) na versão de 15 itens (Yesavage, 1983). No presente estudo são analisadas as seguintes características de pacientes idosos ambulatoriais: sexo, idade, estado civil, escolaridade, presença de acompanhante à consulta, e a pontuação na GDS. Tais informações foram submetidas à análise estatística descritiva, realizada com auxílio do programa SPSS 10.0 for Windows.

\section{Resultados}

Participaram do estudo 303 pacientes atendidos no Ambulatório de Geriatria do Hospital Universitário de Brasilia entre março de 2000 e março de 2001, que haviam sido entrevistados com a Avaliação Geriátrica Objetiva (AGO) durante a consulta médica. A média de idade dos pacientes é de 71 anos $(d p=8,4)$, sendo que um pouco mais da metade da amostra $(n=157 ; 51,8 \%)$ tem entre 60 a 69 anos. A idade média dos idosos do sexo masculino é de 72,4 anos $(d p=7,9)$ e a das mulheres, 70,2 anos $(d p=8,53)$. A Tabela 1 apresenta características relevantes da amostra.

A clientela atendida no Ambulatório de Geriatria é composta primordialmente por pacientes do sexo feminino $(n=204 ; 67,3 \%)$ e com escolaridade até 4 anos $(n=222$; $73,5 \%)$. Desse total, $112(37,1 \%)$ são analfabetos e 110 $(36,4 \%)$ têm de 1 a 4 anos de estudo. Estes resultados são similares para homens e mulheres.

Quanto ao estado civil, grande parte da amostra é casada ( $n=151 ; 49,8 \%)$, seguindo-se os viúvos $(n=92 ; 30,4 \%)$. Há uma diferença digna de nota neste aspecto. Enquanto 70,6\% $(n=70)$ dos homens são casados, sendo viúvos apenas $16,2 \%$ $(n=16)$, as mulheres se dividem primordialmente entre casadas $(n=81 ; 39,7 \%)$ e viúvas $(n=76 ; 37,3 \%)$.

No que se refere ao acompanhante dos pacientes à consulta, encontrou-se que $130(42,9 \%)$ vão sós ao Ambulatório, enquanto 112 (36,9\%) são acompanhados pelos filhos. Não há grande diferença entre as percentagens de homens e mulheres nessas duas categorias. Comparando-se a média de idade dos pacientes desacompanhados com a dos acompanhados ao Hospital pelos filhos tem-se, respectivamente, 65,2 anos e 74,2 anos.

Finalmente, a média de pontuação dos pacientes na Escala de Depressão Geriátrica (GDS) é de 4,8 ( $(p=3,7)$, informação que constava do prontuário de apenas 218 dos 303 idosos. A Tabela 1 disponibiliza resultados dos 218 pacientes avaliados com a GDS. Desse total, 138 $(63,3 \%)$ estão na faixa de 0 a 5 pontos, sugerindo ausência de depressão; por outro lado, 80 pacientes $(36,7 \%)$ obtiveram entre 6 a 15 pontos, o que aponta para depressão moderada (6-10) ou grave (11-15). 
Tabela 1

Características da População Atendida (N=303)

\begin{tabular}{|c|c|c|c|c|c|c|}
\hline \multirow[t]{2}{*}{ Variáveis } & \multicolumn{2}{|c|}{ Mulheres } & \multicolumn{2}{|c|}{ Homens } & \multicolumn{2}{|c|}{ Total } \\
\hline & $n$ & $\%$ & $n$ & $\%$ & $n$ & $\%$ \\
\hline \multicolumn{7}{|l|}{ Idade (anos) } \\
\hline $60-69$ & 116 & 56,9 & 41 & 41,4 & 157 & 51,8 \\
\hline $70-79$ & 56 & 27,5 & 39 & 39,4 & 95 & 31,4 \\
\hline $80-89$ & 26 & 12,7 & 18 & 18,2 & 44 & 14,5 \\
\hline $90-99$ & 6 & 2,9 & 1 & 1,0 & 7 & 2,3 \\
\hline Total & 204 & 100,0 & 99 & 100,0 & 303 & 100,0 \\
\hline \multicolumn{7}{|l|}{ Escolaridade } \\
\hline Analfabeto & 76 & 37,3 & 36 & 36,4 & 112 & 37,1 \\
\hline Até 4 anos & 71 & 34,8 & 39 & 39,4 & 110 & 36,4 \\
\hline 4 a 8 anos & 36 & 17,6 & 16 & 16,2 & 51 & 16,9 \\
\hline 8 ou mais & 16 & 7,8 & 8 & 8,1 & 24 & 7,9 \\
\hline Não consta & 05 & 2,5 & 0 & 0 & 05 & 1,7 \\
\hline Total & 199 & 100,0 & 99 & 100,0 & 297 & 100,0 \\
\hline \multicolumn{7}{|l|}{ Estado Civil } \\
\hline Solteiro(a) & 22 & 10,8 & 7 & 7,1 & 29 & 9,6 \\
\hline Casado(a) & 81 & 39,7 & 70 & 70,6 & 151 & 49,8 \\
\hline Divorciado(a) & 23 & 11,2 & 5 & 5,1 & 28 & 9,3 \\
\hline Viúvo(a) & 76 & 37,3 & 16 & 16,2 & 92 & 30,4 \\
\hline Não consta & 02 & 1,0 & 01 & 1,0 & 03 & 0,9 \\
\hline Total & 202 & 100,0 & 98 & 100,0 & 299 & 100,0 \\
\hline \multicolumn{7}{|l|}{ Acompanhante } \\
\hline Esposo & 11 & 5,4 & 9 & 9,1 & 20 & 6,6 \\
\hline Filho & 73 & 35,8 & 39 & 39,4 & 112 & 36,9 \\
\hline Outros & 29 & 14,2 & 11 & 11,1 & 40 & 13,2 \\
\hline Sem acompanhante & 90 & 44,1 & 40 & 40,4 & 130 & 42,9 \\
\hline Não consta & 01 & 0,5 & 0 & 0 & 01 & 0,4 \\
\hline Total & 204 & 100,0 & 99 & 100,0 & 303 & 100,0 \\
\hline \multicolumn{7}{|l|}{ Pontos GDS $(n=218)$} \\
\hline $0-5$ & 83 & 56,0 & 55 & 78,6 & 138 & 63,3 \\
\hline $6-10$ & 47 & 31,8 & 12 & 17,1 & 59 & 27,1 \\
\hline $11-15$ & 18 & 12,2 & 03 & 4,3 & 21 & 9,6 \\
\hline Total & 148 & 100,0 & 70 & 100,0 & 218 & 100,0 \\
\hline
\end{tabular}

Apresenta-se a seguir diferenças de gênero quanto ao índice de depressão dos idosos. Dos 70 homens submetidos à GDS, 55 (78,6\%) obtiveram entre 0 a 5 pontos (ausência de depressão); os 15 idosos restantes $(21,4 \%)$ encontram-se na faixa de 6 a 15 pontos, indicativa de depressão. A pontuação média masculina na Escala correspondeu a 3,6 $(d p=3,2)$. No que diz respeito às 148 mulheres que responderam à Escala de Depressão Geriátrica, $83(56 \%)$ estão na faixa de 0 a 5 pontos, e 65
$(44,0 \%)$ obtiveram entre 6 a 15 pontos, o que sinaliza a presença de sintomas depressivos. A pontuação média feminina foi de $5,3(d p=3,8)$.

\section{Discussão}

Conforme anteriormente descrito, a maioria dos pacientes atendidos no período de um ano no Ambulatório de Geriatria do HUB têm entre 60 e 69 anos $(m=71)$. 
Quase 70\% dos pacientes atendidos no Ambulatório neste período são mulheres. Mesmo considerando que temos na população brasileira mais idosos do sexo feminino ( $55 \%$ versus $45 \%$, aproximadamente), cabe refletir sobre tal fato. Por que um número mais significativo de homens idosos não chega ao ambulatório? Serão estes de modo geral mais saudáveis que as mulheres, não necessitando assim de cuidados de saúde?

Tal argumento, no entanto, não encontra sustentação nas estatísticas de mortalidade. Não pode ser mais saudável o grupo que morre mais! Ressalte-se, contudo, que a eliminação mais precoce de homens com saúde frágil age como um processo de seleção, fazendo com que os sobreviventes, mesmo em menor número, sejam indivíduos menos vulneráveis que as mulheres como um todo. A menor ocorrência de óbitos precoces no sexo feminino favorece a existência de grande contingente de mulheres vivas, mas com a saúde comprometida, ao lado de outras com excepcional nível de saúde. Dessa forma, os homens, ainda que em menor quantidade, formam um grupo mais homogêneo do que o grupo feminino quanto à saúde.

Investigando idosos residentes no Rio de Janeiro, Veras (1994) encontrou que a proporção de deficiências físicas, cognitivas e depressão era maior entre mulheres. Além disso, a viuvez é maior entre mulheres idosas e estas têm, em geral, nível de instrução mais baixo e renda inferior aos homens. Por outro lado, parece haver uma maior "relutância" do homem - em diversas faixas etárias - a buscar e engajar-se em atividades relativas à prevenção, diagnóstico e tratamento de problemas físicos e emocionais (Robertson \& Fitzgerald, 1992). Blazer e Koening (1999), por exemplo, sugerem que mulheres idosas têm maior probabilidade de admitir e verbalizar seus sentimentos disfóricos que os homens. Estes tendem a negar seus sentimentos ou utilizar recursos como o alcoolismo e tentativas de suicídio.

Em síntese, possivelmente são diversos os fatores associados à diferença na proporção de homens e mulheres no Ambulatório de Geriatria, dado não explicado apenas pela diferença nas taxas de idosos do sexo masculino e feminino na população brasileira Estudos epidemiológicos comunitários podem contribuir para elucidar esta questão, bastante complexa, pode-se dizer. Mulheres, e no caso específico podem queixar-se mais, seja por sentirem mais dores e desconfortos, seja por se permitirem expressar de modo mais intenso suas aflições. Em outras palavras, em comparação com o homem a mulher pode sofrer mais de males físicos e mentais, ou comunicar tal sofrimento de modo mais claro ou veemente que este. Neste último caso, o choro, a lamentação, a procura por médicos, constituem estratégias de certo modo autorizadas e mesmo incentivadas pela nossa cultura à mulher, o que lhe facilita o acesso a profissionais e serviços de saúde.

Tendendo a ser mais difícil para os homens buscar ajuda especializada diante de problemas de natureza física e psíquica, é possível que estes tenham contato com o sistema de saúde em estado mais avançado de doenças. Sendo tal hipótese viável, teríamos tratamentos mais onerosos de problemas mais graves, crônicos ou mesmo irreversíveis, se comparados à assistência que pode ser prestada em nível ambulatorial. Isto é mais verdadeiro, ainda, se entendermos que o acompanhamento geriátrico busca ser integral, e não dirigido à uma especialidade médica apenas, e de caráter preventivo além do curativo.

No que diz respeito ao grau de educação formal, vimos que praticamente $3 / 4$ dos pacientes atendidos tem até 4 anos de estudo, o que configura uma população de baixa escolaridade, muito semelhante para homens e mulheres. É digno de nota o alto grau de analfabetismo entre estes idosos $(37,7 \%)$, índice novamente similar entre os dois sexos. Estes dados podem incluir um viés de seleção sócio-econômica. O Ambulatório de Geriatria, assim como as demais especialidades clínicas do HUB, é freqüentado por idosos mais pobres, entre os quais o analfabetismo é inevitavelmente maior.

Outra informação relevante que este levantamento nos traz diz respeito ao estado civil dos pacientes. Embora grande parte desses sejam casados, quase $1 / 3$ da clientela é viúva. Neste caso temos uma importante diferença, pois enquanto os homens são, em sua maioria, casados, as mulheres se dividem entre casadas e viúvas. Tendo em vista que a perda do parceiro constitui um fator que predispõe à depressão na terceira idade, este fenômeno mulheres viúvas - merece atenção clínica. Até que ponto o índice mais elevado de depressão nas mulheres, de acordo com a GDS, está associado à viuvez?

No entanto, é fundamental que se investigue perdas diversas para além da morte de parceiros, de modo a ampliar nossa compreensão acerca do fenômeno depressivo. Faz-se aqui menção à dimensão afetiva da perda, seja por morte, seja por distanciamento físico ou emocional, de pais, irmãos, esposos, filhos e netos. Tendo em vista que a mulher tradicionalmente assume função de cuidadora, o que muitas vezes se estende ao companheiro, filhos já adultos e possivelmente netos, é razoável supor que tais perdas ou situações graves envolvendo familiares afetem de modo particular à mulher idosa. Neste sentido, sugerimos que se procure 
obter informações sobre perdas significativas no levantamento da história clínica do idoso.

Como vem o idoso à consulta médica? Percebe-se que uma parcela considerável dos pacientes - $43 \%$ - vem só, seguidos daqueles que vêm acompanhados dos filhos. É de certo modo surpreendente que tantos pacientes venham desacompanhados ao Ambulatório. No entanto, encontrou-se uma diferença de idade que merece ser registrada: enquanto pacientes acompanhados pelos filhos têm em média 74,2 anos, os que vêm sozinhos têm em média 65,2 anos, ou seja, tendem a ser nove anos mais novos que os primeiros.

De qualquer modo, os hospitais da rede pública do Distrito Federal situados no Plano Piloto de Brasilia recebem muitos pacientes das Cidades Satélites e do Entorno, realidade que implica para o idoso um tempo considerável de percurso e permanência fora de sua residência e comunidade. Por que, enfim, vem o paciente só ao Ambulatório? Por não ter companhia? Por ser muito cara sua vinda acompanhada ao hospital? Ou por preferir vir só? Embora em geral mais jovens que os pacientes acompanhados pelos filhos, é inegável que a idade de 65 anos traz alguns riscos ao idoso que vem desacompanhado ao hospital.

Apesar de não termos dados para responder com mais precisão a esta questão, cabe investigar se tais pacientes residem sós, ou se não podem contar com a ajuda de parentes ou vizinhos no dia da consulta, diferenciando-os daqueles que vêm sós por opção. Neste último caso, não há como negar o aspecto positivo da autonomia do idoso, de uma certa "independência" no ato de vir só ao hospital. Certamente, mais informações são necessárias para que se tenha uma visão mais abrangente do fenômeno.

Finalmente, quanto à pontuação na Escala Geriátrica de Depressão (GDS), o escore médio da amostra $(4,78)$ sugere a ausência de sintomas depressivos. Entretanto, mais uma vez a comparação entre homens e mulheres aponta diferença digna de nota: enquanto apenas $21,4 \%$ dos primeiros apresentam pontuação indicadora de depressão moderada ou severa, o mesmo ocorre com $44 \%$ das pacientes. Ou seja, mais mulheres sofrem de sintomas depressivos, de acordo com a GDS. Este resultado não foge aos padrões nacionais e internacionais. Pesquisas têm reiteradamente confirmado que mulheres em diferentes etapas da vida sofrem mais de depressão, mesmo quando avaliadas por instrumentos diversos. Esta constatação não nos desobriga de buscar analisar este fenômeno.

Conforme mencionado anteriormente, é preciso lembrar que a viuvez e outras perdas significativas, em especial de familiares, podem contribuir para a depressão entre mulheres idosas. É essencial conhecer mais sobre esta dimensão da vida feminina, bem como sobre os mecanismos psicológicos, sociais e culturais do processo de luto. Entendemos que a morte de filhos deve ser melhor investigada como fator de risco severo à depressão em mulheres, tento em vista que tal perda, seja esta em qualquer período da vida, pode não ser jamais superada por muitas mulheres. Pais, por princípio, não estão preparados para perder seus filhos, ou seja, vêlos partir antes deles próprios, genitores. Tão mais delicada deve ser a situação da mulher, que parece não abdicar ou libertar-se da função materna ao longo de sua vida.

Deve-se igualmente considerar que a velhice na mulher é a continuação de um processo que teve início, pode-se assim dizer, na meia-idade. Não apenas alterações hormonais predispõem a mulher à depressão no climatério, mas igualmente as inúmeras mudanças associadas a esta etapa da vida (Landau, Cyr \& Moulton, 1998). O envelhecimento, embora inevitável, pode representar um ônus adicional para as mulheres, especialmente numa sociedade que lhes exige juventude e beleza.

O fim do período reprodutivo tem significado especial para a mulher, que enfrenta grandes transformações nesta fase, de ordem física, emocional, familiar e social. Separações conjugais, filhos que deixam a familia, aposentadoria pessoal ou do companheiro, são fatores que alteram a vida da mulher e a dinâmica familiar (Greer, 1994; Ramos, 1998). É fundamental, portanto, que sintomas e dificuldades de ordem física e psíquica sejam compreendidos de maneira contextualizada, levando-se em conta características individuais, familiares e sociais daqueles que vêm em busca de ajuda médica e psicológica.

\section{Considerações Finais}

O presente estudo buscou identificar características sóciodemográficas e a pontuação da clientela atendida no Ambulatório de Geriatria do Hospital Universitário de Brasília, Distrito Federal. Visou, ainda, contribuir para o aprimoramento da avaliação clínica de idosos assistidos em serviços ambulatoriais de saúde, tarefa que aproxima clínicos e pesquisadores. Finalmente, espera-se contribuir para ampliar o conhecimento sobre a população idosa que recorre ao sistema público de saúde brasileiro.

Tendo em vista tais objetivos, foram levantadas informações presentes nos prontuários de pacientes atendidos entre março de 2000 e março de 2001. Encontrou-se que os pacientes são, em sua maioria, mulheres, com idade próxima aos 70 anos e poucos anos de estudo. Muitos vêm sós ao ambulatório. É digno de nota que uma parte considerável das idosas (44\%) apresentam indicativo de depressão, de acordo com a Escala de Depressão Geriátrica. 
Tal indicativo merece a atenção de profissionais de saúde, em especial de psicólogos. Estudo subseqüente realizado no mesmo Ambulatório, ainda não-publicado, demostrou a relevância de se investigar a história de vida das pacientes idosas. Vidas marcadas por perdas sucessivas e significativas, o que nos aproxima da compreensão da experiência depressiva.

Neste sentido, entendemos que a avaliação de idosos no contexto hospitalar-ambulatorial deve incorporar dimensões não estritamente médicas, tais como dados significativos da história de vida, mortes e perdas, rede social, ocupação, atividades de lazer, entre outras. Embora não primariamente relacionadas à história médica dos pacientes, tais informações podem trazer indicadores sobre o processo de adoecimento e estratégias de enfrentamento das dificuldades diárias.

Serviços de geriatria em hospitais gerais têm uma função primordial não apenas de tratamento, mas igualmente de prevenção de problemas físicos e psíquicos. Talvez nesta especialidade seja possível exercer um cuidado integral à pessoa. Tal iniciativa pressupõe o trabalho integrado de equipe multidisciplinar, na qual o psicólogo desempenha função relevante. É preciso, deve-se dizer, ousar ir além do atendimento ambulatorial. É nesse contexto que a proposta de Hospitais-Dia deve ser apreciada como uma oportunidade de promoção de saúde e melhoria de vida, para além de simples controle sintomatológico.

Finalmente, no caso específico de Brasília e das Cidades Satélites, devemos considerar que a região tem pouco mais de 40 anos. Os idosos que aqui habitam e vêm em busca de tratamento médico-hospitalar, são provenientes de diversas regiões do Brasil. Neste sentido, atenção especial deve ser dada à migração e suas conseqüências, já que se constitui em fator primordial na determinação da saúde mental dos indivíduos (Ferreira, 1999).

\section{Referências}

Aguiar, W. M. \& Dunningham, W. (1993). Depressão geriátrica: Aspectos clínicos e terapêuticos (Boletim CBPTD). Arquivos Brasileiros de Medicina, 67(Suplemento), 297-298.

Almeida, O. P. (1999). Sintomas psiquiátricos entre paciente com demência atendidos em um serviço ambulatorial. Arquivos de Neuropsiquiatria, 57, $937-$ 943.

Almeida, O. P. \& Almeida, S. A. (1999). Confiabilidade da versão brasileira da Escala de Depressão em Geriatria (GDS): Versão reduzida. Arquivos de Neuropsiquiatria, 57, 421-426.

Almeida, O. P., Garrido, R. \& Tamai, S. (1998). Unidade para Idosos (UNID) do Departamento de Saúde Mental da Santa Casa de São Paulo: Características clínicas de pacientes atendidos em nível ambulatorial. Jornal Brasileiro de Psiquiatria, 47, 291-296.

Associação Psiquiátrica Americana (1995). Manual Diagnóstico e Estatístico dos Transtornos Mentais ( $4^{\mathrm{a}}$ Ed.). Washington, DC.: APA.
Blay, S. L. (1989). Distúrbios psiquiátricos no idoso: Extensão do problema, fatores associados e questões assistenciais. Jornal Brasileiro de Psiquiatria, 38 , 53-54.

Blazer, D. G. \& Koening, H. G. (1999). Transtornos do humor. Em D. G.

Blazer \& E. W. Busse (Orgs.), Psiquiatria geriátrica. Porto Alegre: Artmed. Fernandes, P. (2000). A depressão no idoso; estudo da relação entre fatores pessoais e situacionais e manifestações da depressão. Coimbra: Quarteto.

Ferreira, A. P. (1999). O migrante na rede do outro: Ensaios sobre alteridade e subjetividade. Rio de Janeiro: Te Corá.

Greer, G. (1994). Mulher: Maturidade e mudança. São Paulo: Augustus.

Guimarães, R. M. (1996). É possível retardar o envelhecimento? Em M. L. Guidi \& M. R. Moreira (Orgs.), Rejuvenescer a velhice (pp. 17-22). Brasília: Editora da UnB.

Guimarães R. M., Freire Neto, J. B., Costa, E., Silva, E. A. \& Moura E. B. (1997). Depressão: A morbidade oculta em pacientes idosos cirúrgicos. Brasília Médica, 34, 5-14.

Landau, C., Cyr, M. G. \& Moulton, A. W. (1998). O livro completo da menopausa: Guia da boa saúde da mulher. Rio de Janeiro: José Olympio.

Linhares, C. (2002). Histórias de vida: Contribuições acerca da experiência depressiva nos anos tardios de vida. Dissertação de Mestrado não-publicada, Curso de PósGraduação em Psicologia, Universidade de Brasilia. Brasília, DF.

Neri, A. L. (1995). Psicologia do envelhecimento: Uma área emergente. Em A. L. Neri (Orgs.), Psicologia do envelhecimento (pp. 13-40). Campinas, SP: Papirus.

Papalia, D. \& Olds, S. (2000). Desenvolvimento bumano. Porto Alegre: Artes Médicas. Ramos, D. (1998). Viva a menopausa naturalmente. São Paulo: Augustus.

Robertson, J. M. \& Fitzgerald, L. F. (1992). Overcoming the masculine mystique: Preferences for alternative forms of assistance among men who avoid counseling. Journal of Counseling Psychology, 39, 246-249.

Stoppe Jr., A., Jacob Filho, W. \& Louzã Neto, M. (1994). Avaliação de depressão em idosos através da Escala de Depressão em Geriatria: Resultados preliminares. Revista ABP-APAL, 16, 149-153.

Stoppe Jr., A. \& Louzã Neto, M. (1999). Depressão na terceira idade: Apresentação clinica e abordagem terapêutica. São Paulo: Lemos.

Taddei, C., Ramos, L. R, De Moraes, Wajngarten, A., Santos, S., Savioli, F. \& Dioguardi, R. (1997). Estudo multicêntrico de idosos atendidos em ambulatórios de cardiologia e geriatria e instituições brasileiras. Arquivos Brasileiros de Cardiologia, 69, 329-333.

Veras, R. P. (1994). País jovem com cabelos brancos: A saúde do idoso no Brasil. Rio de Janeiro: Relume.

Yesavage, J., Brink, T., Rose, T., Lun, O., Huang, V., Adey, N. \& Leirer, V. (1983). Development and validation of a geriatric depression scale: A preliminary report. Journal of Psychiztric Research, 17, 37-39.

World Health Organization (1992). The ICD-10 Classification of mental and behavioural disorders: Clinical Discriptions and diagnostic headlines. Geneve: WHO.

Yessavage, J. A. (1988). Geriatric depression scale. Psychopharmacology Bullettin, 24,709 . 
Sobre os autores

Cristina Ramos Costa Linhares é Psicóloga, Mestre em Psicologia Clínica pela Universidade de Brasília.

Vera Lúcia Decnop Coelho é Psicóloga, Doutora em Psicologia pela Case Western Reserve University. É Professora do Departamento de Psicologia Clínica da Universidade de Brasília.

Renato Maia Guimarães é Médico Geriatra, Diretor do Centro de Medicina do Idoso do Hospital Universitário de Brasília, da Universidade de Brasília.

Ana Paula Martins de Campos é Psicóloga, Mestranda do Programa de Pós-graduação em Psicologia da Universidade de Brasília.

Nális Torres de Carvalho é Graduanda em Psicologia da Universidade de Brasília. 